\title{
LTE-Advanced Based Handover Mechanism for Natural Disaster Situations
}

\author{
Sayan Kumar Ray ${ }^{1}$, Nurul I Sarkar ${ }^{2}$, Devatanu Deka ${ }^{3}$ and Swapan Kumar Ray ${ }^{4}$ \\ ${ }^{1}$ Faculty of Business and Information Technology, Manukau Institute of Technology, Auckland, New Zealand \\ ${ }^{2}$ School of Computer and Mathematical Sciences, Auckland University of Technology, Auckland, New Zealand \\ ${ }^{3}$ Dynamic Controls, Christchurch, New Zealand \\ ${ }^{4}$ Dept of Computer Science and Engineering, Jadavpur University, Kolkata, India
}

sayan.ray@manukau.ac.nz,nurul.sarkar@aut.ac.nz,deva.deka@gmail.com,skray@ieee.org

\begin{abstract}
Telecommunication networks often face power outage problems in the natural disaster affected areas. Also, owing to a sudden substantial increase in network traffic loads the battery backup power of the base stations run out quickly and therefore hampering telecommunication services. To overcome this system performance issues, we propose a Long Term Evolution (LTE)Advanced (LTE-A)-based user equipment (UE)-controlled and base station (Evolved Node B or eNB)-assisted handover scheme. The idea is to limit the arrival of new traffic to an already overloaded eNB by diverting their handover to lightly loaded nearby eNBs. The novelty of this work is the ability of an UE to self-detect the occurrence of a natural disaster and to self-select the most suitable target eNB (TeNB) to handover with in the disaster affected areas. The handover is performed by obtaining the weighted average score (WAS) of the direction of motion (DoM) and the leftover battery backup power of the different neighboring eNBs (NeNB). The UE also predicts its DoM and dynamically adjust the weights of the two parameters if it's a disaster situation. Preliminary simulation results show that the scheme can offer up to $65 \%$ handover success rate in disaster situations.
\end{abstract}

Keywords-natural disaster, seamless handover, leftover power, base station, LTE-A, weighted average score, movement prediction

\section{INTRODUCTION}

Telecommunication networks have become an important part of our daily lives because people would be immobilized without network and telecommunication services. They are expected to be stable and reliable always. However, natural disasters, like earthquakes and hurricanes, may damage the whole network infrastructure or cause breakdown of the power grid in the affected areas hampering telecommunication services [1-2]. Immediately after a natural disaster the telecommunication networks become heavily congested as a result of sudden and huge increase in network traffic/calls in the affected areas. This results to a complete collapse of the whole network coverage as eNode B (eNB or base station) battery backup dies down very quickly. A practical example of this disaster situation is the earthquake in Christchurch, New Zealand in 2011. Network traffic overloading of the eNBs in the affected areas can be caused by (a) the sudden substantial increase in voice calls to and from people stuck in the affected areas; and (b) the addition of new communication traffic generated by the rescue and relief team members and other people arriving for rendering help. The network outage occurs owing to power outage and traffic congestion. In such situations, it is always helpful if the battery backups in the affected eNBs sustain for longer time enabling people in need to carry on essential communications.

To overcome the above problems, we propose an UEcontrolled handover scheme for natural disaster scenarios. We consider LTE-A as an underlying network architecture. The key idea is that an UE self-detects the occurrence of disasters and then self-selects the best TeNB for handover using selfpredicted DoM and the leftover power (LoP) of the battery backups of the NeNBs in the affected areas (The concept of LoP is explained in Section III). The UE assigns scores to each NeNB against these two parameters and finally selects the TeNB based on the highest weighted average score (WAS). This work is an extension to our previous work published in [2]. The proposed scheme aims at discouraging the arrival of new traffic/calls to an already overloaded natural disaster affected eNB in order to prolong the overall lifetime of the battery backup in the eNBs. This in turn would prolong the flow of the number of voice calls and text messages immediately after a disaster situation by utilizing the LoP of the backup batteries before they die down. We used a relative velocity-based DoM prediction algorithm which is different to the angle-of-divergence based prediction method used in [2]. We also propose a novel mechanism of an UE self-detecting the natural disaster situation and dynamically adjusting the weighted values of the DoM and LoP. The proposed handover scheme offers the following three main benefits during natural disasters. First, existing connections continue to receive good QoS. Second, new connections may be diverted to lessoverloaded nearby NeNBs. Finally, residual battery life of eNBs is considerably prolonged. The remaining paper is organized as follows. Section II reviews related works. The proposed handover scheme is discussed in Section III. Simulation results are presented in Section IV and a brief discussion in Section $\mathrm{V}$ ends the paper. 


\section{RELATED WORK}

The fact that cellular eNBs in the disaster affected areas die down quickly because of massive network traffic congestion after facing power outage is becoming a critical public safety issue. eNBs at local cell sites have battery backups, which may last for up to 8 hours depending on traffic loads at the site [3- 4]. The number of cell phone call requests immediately after a natural disaster incident shoots up to 8 to 10 times more than during normal situations [5]. In recent times, multiple counter mechanisms are proposed that mostly aim at increasing the backup power capacity in base stations. These include the use of fuel cells and hydrogen back up power supply as well as providing base stations with powerful generators that are able to sustain longer [6-8]. A robust never die network (NDN) constituting of self-powered fixed base stations, cognitive mobile base stations and wireless balloon stations is proposed in [5]. The sudden substantial spikes in network traffic load in the disaster areas overwhelm the transmission capabilities of the network resulting in a lack of spectrum availability for communication. To counter such situations, an emergency communication cognitive radio vehicular (EC-CRVN) architecture is proposed in [9] that may serve as the backbone for essential communications in the affected areas. This network senses for currently unused frequencies in the wireless spectrum licensed to other operators and used those for essential communications. [10] proposes a mechanism of on-the-fly establishment of multihop wireless access networks (OEMAN) for disaster response. The purpose of such an initiative is to extend Internet connectivity from surviving WiFi access points in the affected areas to disaster victims using their own mobile devices.

\section{THE PROPOSED HANDOVER SCHEME}

The proposed scheme is based on an LTE-A UE-controlled and eNB-assisted handover applicable to areas affected by natural disasters such as earthquakes and tornadoes. In this scheme an LTE-A UE (e.g., a smartphone) detects a natural disaster situation, dynamically assigns weights to key parameters such as DoM and LoP and finally selects the best TeNB for handover. The selection of TeNB is based on the combination of two independent parameters, namely, the UE's DoM prediction (the UE self-tracks its movement direction) relative to its NeNBs and the LoP of the battery backups of different NeNBs. The UE assigns scores on the DoM $\left(\mathrm{S}_{\mathrm{DoM}}\right)$ and the $\mathrm{LoP}\left(\mathrm{S}_{\mathrm{LoP}}\right)$ to individual NeNB and the selection of the TeNB for handover activity is based on the highest WAS obtained by an NeNB. The UE thus avoids selecting any NeNB with very little LoP for communication and can thereby maintain a satisfactory QoS for an ongoing traffic call even after the handover. A relative velocity-based DoM prediction mechanism for UE is used. The novel mechanism of LoP estimation of an eNB battery backup is also used in this work [2]. In an LTE-A network, an UE may receive the LoP information of the different NeNBs from the SeNB either as an added field in the existing Handover Measurement Control Request message or as an emergency broadcasted information service similar to the newly introduced 3GPP Earthquake and Tsunami Warning Service (ETWS) and Commercial Mobile Alert Service (CMAS) [11-12].

In addition to the above contributions, we also propose a novel technique for UE to self-detect natural disasters and dynamically adjust the weights of the two parameters, DoM and LoP, as needed. This dynamic allocation of weights based on the underlying environment is more realistic than allocating fixed weights and changing them manually as proposed in [2], where we have assigned some non-zero weights to $\mathrm{S}_{\mathrm{LoP}}$ even during the normal non-disaster situations. The parameter LoP is relevant and meaningful only at the time of natural disaster. On the contrary, the DoM is relevant at all times. Thus, the score $\mathrm{S}_{\mathrm{LoP}}$ of LoP should come into play and be given a weight only at the time of the actual onslaught of a natural disaster and certainly not at other times. Giving non-zero weights to $\mathrm{S}_{\mathrm{LoP}}$ at other times would hamper the quality of the handover. This is because at normal situation the DoM should control the handover solely and must receive $100 \%$ weightage. Giving any weightage to $\mathrm{S}_{\mathrm{LoP}}$ during non-disaster situation is of no use and may add meaningless and random scores in the computation of $\mathrm{S}_{\mathrm{WAS}}$, which eventually may distort the vital DoM score leading to a wrong choice of TeNB for handover during non-disaster situations. Thus in achieving a good handover performance, $\mathrm{S}_{\mathrm{LoP}}$ from NeNBs should be used only during the times of actual onslaughts of natural disasters, whereas, $\mathrm{S}_{\mathrm{DoM}}$ should be computed at all times. To summarize, during non-disaster situations the handover should be carried out solely based on $\mathrm{S}_{\mathrm{DoM}}$. In this paper we have assigned a weight of $50 \%$ to $\mathrm{S}_{\mathrm{LoP}}$ for disaster situations only.

The overall scheme has three different parts: (a) UE assigning individual scores $\left(\mathrm{S}_{\mathrm{DoM}}\right)$ to the NeNBs depending on its direction of motion, (b) UE assigning scores $\left(\mathrm{S}_{\mathrm{LoP}}\right)$ to NeNBs based on their leftover battery power and (c) UE selfdetecting a disaster situation and automatically adjusting the weights of DoM and LoE accordingly and calculating the WAS to select the final TeNB for handover. The detailed handover scheme is presented next.

\section{A. Relative Velocity-based DoM Prediction Scheme}

We employ a distance estimation and lookahead-based handover scheme to predict the UE's DoM relative to its NeNBs [13]. According to this concept, an UE approximately estimates its current distance, from the SeNB as well as from its NeNBs, by monitoring the received signal strength (through periodic measurements/scanning) from the eNBs concerned. For estimating the distance, the UE uses any suitable path loss property of the communication channel. Using a set of at least two distance estimates for each NeNB, a UE can self-track its DoM via simple computation of its relative velocity with respect to each NeNB [13-14]. These estimates allow the UE to look ahead and to determine the $\mathrm{NeNBs}$ to continue/discontinue monitoring (i.e., scanning) and, most importantly, which NeNB the UE is likely to come closer after it leaves its current cell. This knowledge will, in effect, allow the UE to make the best choice of the potential TeNB (among all the NeNBs being scanned/measured) to which it should be handed over by the SeNB. We assume that 
the UE's motion, while it is at the fag end of its journey across a cell, is "broadly linear" over a certain time frame.

In the proposed method, the UE does multiple distance calculations, periodically, for each of the NeNB and from those set of distance samples it can self-ascertain whether its movements relative to a particular NeNB is progressive or regressive. The UE can then compare the progressive movements to identify the NeNB with the highest accumulated forward movement (AFM). Basically, the proposed scheme operates as follows. From the chosen most appropriate path loss Equation, the UE can easily get an estimate of its distance from any particular NeNB at different points in time. Thus, if its distance $\mathrm{d}$ to an NeNB is estimated as $\mathrm{d}_{1}=\mathrm{d}\left(\mathrm{t}_{1}\right)$ and $\mathrm{d}_{2}=\mathrm{d}\left(\mathrm{t}_{2}\right)$ at the time instants $\mathrm{t}_{1}$ and $\mathrm{t}_{2}\left(\mathrm{t}_{2}>\mathrm{t}_{1}\right)$, respectively, then, during the duration $T=t_{2}-t_{1}$ of the time interval $\left(t_{1}, t_{2}\right)$, the MS has an average relative velocity of

$\bar{v}_{1,2}=\left(\mathrm{d}_{2}-\mathrm{d}_{1}\right) / \mathrm{T}=\left|\bar{v}_{1,2}\right| \operatorname{sgn}\left(\bar{v}_{1,2}\right)$

with respect to the NeNB, where $\bar{v}_{1,2}$ is a simpler representation for $\bar{v}_{t 1, t 2}$ (i.e. the average relative velocity of the UE with respect to the NeNB during the time interval $\mathrm{T}=\mathrm{t}_{2}-\mathrm{t}_{1}$.

In Eq. 1, the magnitude $\left|\bar{v}_{1,2}\right|$ of $\bar{v}_{1,2}$ indicates how fast the $\mathrm{UE}$ is approaching towards or receding from the NeNB, i.e., $\left|\bar{v}_{1,2}\right|$ indicates the speed of progression or regression of the UE, relative to the NeNB. On the other hand, sgn $\left|\bar{v}_{1,2}\right|$ signifies whether the UE is moving towards [if $\operatorname{sgn}\left|\bar{v}_{1,2}\right|<0$ ] or away from $\left[\operatorname{sgn}\left|\bar{v}_{1,2}\right|>0\right]$ the NeNB, implying thereby whether the motion of the UE, relative to the $\mathrm{NeNB}$, is progressive or regressive. It is obvious that if the motion of the UE relative to a particular NeNB is regressive, (i.e. $\operatorname{sgn}\left|\bar{v}_{1,2}\right|>$ 0 ), then that NeNB should not be considered as a potential TeNB by the UE. Thus, an UE basically chooses its TeNB based on the acquisition of a few periodic samples of the RSS from each NeNB and then use of the principle of selfestimation of distance followed by a simple lookahead scheme. We keep all the successive sampling periods (i.e. the inter-scanning intervals) constant at $\mathrm{T}$ seconds, i.e. if $\mathrm{T}=\mathrm{t}_{\mathrm{i}}-\mathrm{t}_{\mathrm{i}-1}$ for all $\mathrm{i}, \mathrm{i}=2,3, \ldots$, and assume that $\left\{\mathrm{d}_{\mathrm{i}}\right\}$ are the distances estimated at the scanning instants $\left\{\mathrm{t}_{\mathrm{i}}\right\}, \mathrm{i}=1,2, \ldots$. This makes the values $\left\{\Delta_{\mathrm{i}-1, \mathrm{i}}\right\}=\left\{\mathrm{d}_{\mathrm{i}}-\mathrm{d}_{\mathrm{i}-1}\right\}$ (i.e. $\Delta_{1,2}=\mathrm{d}_{2}-\mathrm{d}_{1}, \Delta_{2,3}=\mathrm{d}_{3}-\mathrm{d}_{2}$, and so on) of the successive "differences in consecutive distances" of the UE from an NeNB themselves represent the average velocity (after scaling by the factor $1 / \mathrm{T}$ ) of the UE, relative to the NeNB, during the respective equal time intervals $\left(t_{1}, t_{2}\right)$, $\left(\mathrm{t}_{2}, \mathrm{t}_{3}\right)$ and so on. Accordingly, each individual "differences in consecutive distances" may, generally speaking, be given by the following vector:

$\Delta_{\mathrm{i}-1, \mathrm{i}}=\mathrm{d}_{\mathrm{i}}-\mathrm{d}_{\mathrm{i}-1}=\left|\mathrm{d}_{\mathrm{i}}-\mathrm{d}_{\mathrm{i}-1}\right| \operatorname{sgn}\left(\mathrm{d}_{\mathrm{i}}-\mathrm{d}_{\mathrm{i}-1}\right)$

To illustrate this direction of motion detection scheme, we consider the scenario depicted in Figure 1 [13]. We assume that the UE measures the RSS from each NeNB at a set of chosen time instants through scanning. In the figure, the UE has four NeNBs, B, C, D and E, clustered around its SeNB A and the UE is moving along a straight line (shown in the solid line) in the direction of the arrow. Thus, referring to Eq. (2), the consecutive distance differences of the UE from an NeNB (say NeNB B), in Figure 1, if scanned during the time interval $\left(t_{i-1,1} t_{i}\right)$, will be given by

$\Delta_{\mathrm{i}-1, \mathrm{i}}(\mathrm{B})=\mathrm{d}_{\mathrm{i}}(\mathrm{B})-\mathrm{d}_{\mathrm{i}-1}(\mathrm{~B})=\left|\mathrm{d}_{\mathrm{i}}(\mathrm{B})-\mathrm{d}_{\mathrm{i}-1}(\mathrm{~B})\right| \operatorname{sgn}\left[\mathrm{d}_{\mathrm{i}}(\mathrm{B})-\mathrm{d}_{\mathrm{i}-1}(\mathrm{~B})\right]$

Initially, we make the assumption that the UE is presently enjoying satisfactory signal strength from its current SeNB A. On detecting the drop in its RSS from SeNB, the UE decides to go for the handover and starts measuring (scanning) each of the NeNBs at T second intervals. The number of measurement cycles and $\mathrm{T}$ are chosen based on factors such as the current velocity of the UE, the number of NeNBs, etc. For simplicity, we assume that the MS initiates three consecutive scanning cycles at the time instants $t_{1}, t_{2}$ and $t_{3}$, where $t_{2}-t_{1}=t_{3}-t_{2}=T$. The UE is located at the points $\mathrm{a}, \mathrm{b}$ and $\mathrm{c}$, respectively, on the line of its motion, at these time instants (refer to Fig. 1). During each of the scanning cycles, the UE acquires its distance estimates from all the four NeNBs B, C, D and E. Thus it obtains the three sets of approximate distances $\{\mathrm{aB}$, $\mathrm{aC}, \mathrm{aD}, \mathrm{aE}\},\{\mathrm{bB}, \mathrm{bC}, \mathrm{bD}, \mathrm{bE}\}$ and $\{\mathrm{cB}, \mathrm{cC}, \mathrm{cD}, \mathrm{cE}\}$ at approximately the three successive $\mathrm{T}$ second intervals beginning $t_{1}, t_{2}$ and $t_{3}$. Next, referring to Equations 2-3, the UE computes the " $\Delta_{\mathrm{i}-1, \mathrm{i}}$ " with respect to each of the four NeNBs (i.e. B, C, D and E in Figure 1) at time $t_{2}$, at the end of the first inter-scanning interval $\left(\mathrm{t}_{1}, \mathrm{t}_{2}\right)$ as $\Delta_{1,2}(\mathrm{~B})=\mathrm{bB}-\mathrm{aB}, \Delta_{1,2}(\mathrm{C})=$ $\mathrm{bC}-\mathrm{aC}, \Delta_{1,2}(\mathrm{D})=\mathrm{bD}-\mathrm{aD}$ and $\Delta_{1,2}(\mathrm{E})=\mathrm{bE}-\mathrm{aE}$. Similar results are obtained for the next inter-scanning interval $\left(\mathrm{t}_{2}, \mathrm{t}_{3}\right)$. At the end of the required number of measurement cycles (let's say for 3 cycles in this case), the UE simply accumulates its relative movement samples with respect to each NeNB and computes the respective 'accumulated forward movement' (AFM), during the entire scanning session for each $\mathrm{NeNB}$ (B, C, D and E) as $\mathrm{AFM}_{\mathrm{B}}: \Delta_{1,2}(\mathrm{~B})+\Delta_{2,3}(\mathrm{~B}), \mathrm{AFM}_{\mathrm{C}}: \Delta_{1,2}(\mathrm{C})+$ $\Delta_{2,3}(\mathrm{C}), \mathrm{AFM}_{\mathrm{D}}: \Delta_{1,2}(\mathrm{D})+\Delta_{2,3}(\mathrm{D})$ and $\mathrm{AFM} \mathrm{E}_{\mathrm{E}}: \Delta_{1,2}(\mathrm{E})+\Delta_{2,3}(\mathrm{E})$. It should be noted here that each term as well as each of the AFM values may be either positive or negative. Based on the calculated AFMs, the UE assigns DoM scores (SDoM) to each

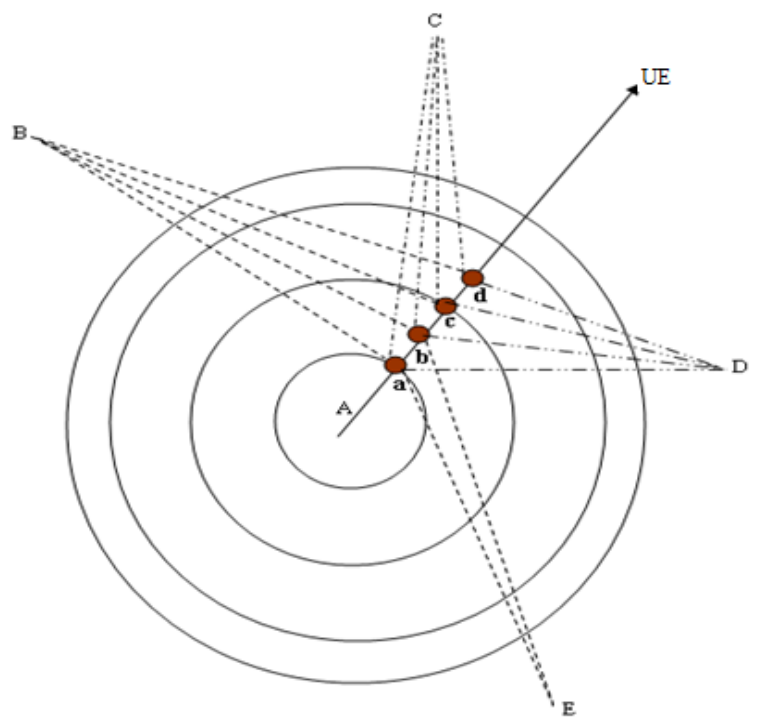

Fig. 1: UE's movement direction prediction scheme 
of the individual NeNBs and the NeNB with the maximum AFM value gets the highest score (as the UE shows maximum progressive movement with respect to this NeNB; in Fig. 1 its NeNB C) and that with the minimum value get the lowest score.

\section{B. LoP-based Scoring and WAS Calculation}

In the aftermath of a natural disaster, most often the eNBs in the affected areas loose electrical power from the underlying grid because of an outage. Owing to that they switch to either the backup battery power or the generator power to sustain. However, because of the sudden heavy increase in traffic load the backup power in eNBs does not last long either and the whole mobile network is jammed owing to congestion until those eNBs die down. For such situations we assume that eNBs keep track of their leftover backup power and have the intelligence to self-estimate (even approximately) how long that may last based on the existing traffic load situation [2]. In an LTE-A network, the eNBs are able to exchange this information through the backbone X2 interfaces. A "X2 Power Indication" message is proposed that can be exchanged amongst each other over the X2 interface by the different eNBs [2]. Such a message can be particularly very useful for eNBs in disaster affected areas to inter-exchange leftover power information.

A novel scheme of estimating the LoP of a backup battery in an eNB was proposed by us in [2]. In this context, a simple explanation of the concept of LoP is provided here. Conceptually, LoP is the difference between the maximum instantaneous power that can be delivered by a battery without being overloaded and the average instantaneous power that is being actually delivered by the battery. A large value of LoP indicates that the battery is capable of delivering much more power than what it is delivering presently, which implies that an eNB can invite more connections. Practically speaking, LoP should be measured over a small interval. Accordingly, from a practical perspective, LoP may be defined as "the difference between the average maximum power that can be delivered by a battery during small interval without being overloaded and the average power that is actually being delivered by the battery during the same small interval".

On receiving the LoP information about different NeNBs from its $\mathrm{SeNB}$, the UE assigns them individual scores $\left(\mathrm{S}_{\mathrm{LoP}}\right)$ and the NeNB with the least LoP gets the lowest score. The details of this LoE-based score assignment technique can be found in [2]. It is well known that the number of connections (voice and data) currently handled by an eNB, provides an indirect and approximate measure of the traffic load it is handling. Thus there is a direct proportionality between the number of connections being currently handled by the eNB and the traffic load on the eNB [15]. This observation gave us a clue towards making an approximate estimation of the LoP of the battery at any time. We assumed that an eNB has a capacity of handling $\mathrm{N}$ connections at any time. This implies that the eNB can support a maximum of $\mathrm{N}$ connections at any time without lowering the QoS enjoyed by the connections, with the battery voltage still maintaining its rated (maximum) value and the supplied battery power being the maximum. We assumed ' $n$ ' to be the number or count of connections that are currently being handled by the eNB. There is a direct proportionality between ' $n$ ' and the eNB's present call traffic power consumption, i.e., traffic load. Also, $\mathrm{N}>=\mathrm{n}$ at any time. Allowing the eNB to handle more than ' $n$ ' connections at any time, will basically overload its battery causing noticeable deterioration in the QoS of the calls. The LoP of the battery of an eNB equals $[(\mathrm{N}-\mathrm{n}) / \mathrm{N}] *$ Rated power of the battery. Accordingly, under this particular condition of the traffic load (with ' $n$ ' active connections), the score assigned to the eNB, against the LoP of its battery, is given by $\mathrm{S}_{\mathrm{LoP}}=(\mathrm{N}$ n) / N. So, obviously, the more loaded an eNB is, the fewer the number of new connections it invites to pass through it and less score it gets.

Once the UE has scored all the NeNBs against the DoM and LoP parameters, it calculates the WAS for each of the NeNB and the NeNB receiving the highest WAS is selected by the UE as the TeNB to handover with. However, for the scheme proposed in [2] the weights are manually assigned to the DoM and LoP parameters without taking much into consideration the underlying environment condition. Here we propose a more realistic mechanism of dynamically adjusting the parametric weights according to the underlying condition.

\section{Dynamic Weight Assignment by an UE}

In order to achieve a good handover performance at all times we propose that an UE needs to self-assess the underlying environment condition and self-detect the actual beginning of the onslaught of a natural disaster as an event. Depending on the condition, an UE can then change the assigned weights dynamically as and when needed with no requirement of any manual intervention. We propose a scheme which basically requires the detection of the actual beginning of the onslaught of a natural disaster as an event. Before the occurrence of this event, i.e., at normal times, the weight of the parameters should be initialized as $\mathrm{W}_{\mathrm{DoM}}=1.0$ and $\mathrm{W}_{\mathrm{LoP}}=$ 0.0. This is because the parameter LoP is relevant and meaningful only at the times of natural disasters and not otherwise. However, immediately after the detection of a natural disaster event, the weights should be set as $\mathrm{W}_{\mathrm{DoM}}=0.5$ and $\mathrm{W}_{\mathrm{LoP}}=0.5$. Finally, after the natural disaster subsides, the weightage should be reset to their normal values, i.e., $\mathrm{W}_{\mathrm{DoM}}=$ 1.0 and $\mathrm{W}_{\text {LoP }}=0.0$.

UE will detect a natural disaster event and switch the weights accordingly in the following way. In context to the LoP scoring procedure discussed in the previous section, it is observed that although during normal times, the value of ' $n$ ' increases and decreases randomly with time (depending on eNB traffic loads) at a relatively slow rate, it always remains below the eNB capacity $\mathrm{N}$ (i.e., $\mathrm{N}>\mathrm{n}$ ). On the contrary, during the beginning of the onslaught of a natural disaster, ' $n$ ' tends to monotonically increase and that too at a much higher rate than at the normal times. Thus the event of the beginning of the onslaught of a natural disaster can be easily detected by periodically sampling the variable number ' $n$ ' of the count of connections in a eNB and accumulating the count values up to few samples (for example, let's say, four samples). Although, during normal times, the accumulation of count samples will 
be relatively small, at the beginning of the onslaught of a natural disaster, the accumulation of connection count samples will be much larger. At this point, we assume that the sampling is always done every $T_{S}$ seconds (in practice, $T_{S}$ can be around 1 to 2 minutes), to generate the sequence of connection count samples $\mathrm{n}(\mathrm{t}), \mathrm{n}\left(\mathrm{t}+\mathrm{T}_{\mathrm{S}}\right), \quad \mathrm{n}\left(\mathrm{t}+2 \mathrm{~T}_{\mathrm{S}}\right)$, $\mathrm{n}\left(\mathrm{t}+3 \mathrm{~T}_{\mathrm{S}}\right), \ldots \ldots \ldots, \mathrm{n}\left[\mathrm{t}+(\mathrm{p}-1) \mathrm{T}_{\mathrm{S}}\right], \mathrm{n}\left(\mathrm{t}+\mathrm{p} \mathrm{T}_{\mathrm{S}}\right), \mathrm{n}\left[\mathrm{t}+(\mathrm{p}+1) \mathrm{T}_{\mathrm{S}}\right], \ldots$, etc. In this context, it should be noted that LoP of an eNB only changes over a time frame of minutes, not seconds as much of the LoP calculation depends on the present load of an eNB, which only changes over minutes [13]. Hence the value of the accumulated count of samples $M$ at the time $\left(\mathrm{t}+\mathrm{pT} \mathrm{T}_{\mathrm{S}}\right)$ may be given by

$$
\mathrm{M}=\sum_{\mathrm{i}=\mathrm{p}-3}^{\mathrm{i}=\mathrm{p}} \mathrm{n}\left(\mathrm{t}+\mathrm{iT}_{S}\right)
$$

When the accumulated count of samples $M$ will exceed an appropriately chosen threshold value, the desired event will be considered to have occurred and, accordingly, UE will automatically set the weights of the parameters to $\mathrm{W}_{\mathrm{DoM}}=0.5$, $\mathrm{W}_{\mathrm{LoP}}=0.5$. Then, the UE will calculate the WAS and choose the highest value NeNB as the TeNB for handover. Similarly, after natural disaster subsides, the weights will be reset to $\left(\mathrm{W}_{\mathrm{DoM}}=1.0, \mathrm{~W}_{\mathrm{LoP}}=0.0\right)$. Both these setting and resetting of the weights will be carried out through program interrupts.

\section{Simulation Model VALIDATION}

A Python-based LTE-A simulator has been developed to validate the proposed handover mechanisms. We primarily aimed at validating the system model ensuring that the proposed scheme is resulting in the right choice of the TeNB based on the two parameters, DoM and LoP, as per the algorithm. In the simulation topology, 400 cells are considered in a $20 \times 20$ square array, with each cell having one eNB in it. A $500 \mathrm{~m}$ of inter-eNB distance is considered. The simulation runs on a flat terrain, where movements are defined in steps of $10 \mathrm{~m}$. Also, the distance between two grid lines is arbitrarily assumed to be $10 \mathrm{~m}$. Coverage overlap exists between adjacent eNBs in the simulation terrain and all eNBs are assumed to be connected to the backbone network. We assume that each eNB has eight NeNBs around it (except the ones at the boundary of the terrain) and each of them is aware of its location in the terrain.

We considered simulating the movements of UEs in different situations where the user is moving (i) along the motorways or the state highways with the roads being relatively straight and not zigzag or random, (ii) in the cities with the roads/movements being straight/curvy/zigzag but not random; and (iii) in the city centre where the roads are laid out in the form of grids. We, thus, considered three different mobility models, namely, the Random Waypoint model, the Random Direction model and the Manhattan model, to simulate the realistic movements of the UE. In accordance to the underlying mobility model, the UE randomly moves through different paths and performs multiple handovers. For every path of the UE, we have tracked its movement carrying out multiple successive handovers with different NeNBs (one of these NeNBs after handover becomes the successive SeNB for the UE). For every handover the UE carries out, the simulation works as per the explanations in Section IV to compute the individual scores of NeNBs. The WalfischIkegami model [14] is implemented to simulate the path loss behaviours of the UE. Every eNB in the terrain is assigned a load value and the LoP is estimated based on that. As primarily we aim to validate the reliability of this scheme, i.e., whether the UE is selecting the right TeNB according to our scheme, we have recorded whether the eNBs with which handovers are actually performed, match the eNBs as per the prediction of our scheme (in that case we call it a 'correct' or reliable handover) or not (an 'incorrect' handover). The results presented here are based on the method of multiple independent replications each of which continued until the UE stopped its movements at the end of the time frame for each simulation run.

Preliminary simulation results presented in Fig. 2 show the percentage of correct handovers for our proposed scheme against the UE's movement paths following the three different mobility models. $\mathrm{S}_{\mathrm{WAS}}$ for each $\mathrm{NeNB}$ is calculated against two weight ratios assigned dynamically to the two parameters by the UE depending on the underlying environment condition, i.e., non-disaster or disaster situation. For the former the DoM:LoP weight ratio is 100:0, while the weight ratio for the latter is 50:50 as discussed in Section III. As shown in Figure 2, the percentage of correct number of handovers is always better during a non-disaster situation compared to a disaster situation. This is true for all the three mobility models and is primarily due to the fact that eNB LoP, which is estimated based on the random eNB load values, is not assigned any weightage during a non-disaster situation and DoM received $100 \%$ weightage value. Of the three mobility models, Manhattan model offered the highest handover success rate both in non-disaster and disaster situations. The success rate during non-disaster situations is more than $89 \%$ and around $65 \%$ if there is a disaster. Compared to this, the handover success rates for the other two mobility models are much less. This is due to the square grid like nature of the Manhattan Model corresponding to the field also being arranged in a square grid. A better prediction is made for motion in 45 degree intervals than any other angle as the grids of eNBs naturally form these angles themselves. Also, while choosing the next random direction to move, in case of the Manhattan model, where the roads are in the form of grids, the UE just has to choose one random direction out of only four different directions available to choose from. So, the time taken to make each of these choices is shorter than the time taken in case of the other two mobility models.

\section{CONCLUSIONS}

In this paper we proposed an LTE-A based UE-controlled and eNB-assisted handover mechanism suitable for communication in the natural disaster scenarios. The proposed mechanism prolongs the overall lifetime of the battery backups in the affected eNBs so that the communication network could work little longer enabling the people stuck in the affected areas to make some urgent calls. Simulation 


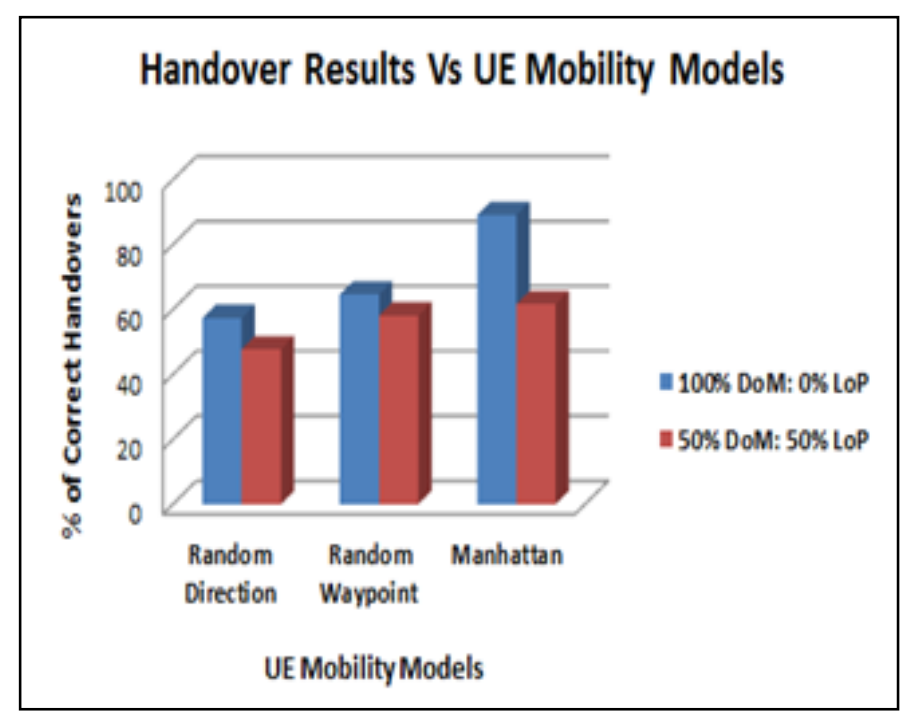

Fig. 2. Handover Results for Selection of TeNB

studies have shown that the proposed scheme can offer up to $65 \%$ handover success rate in disaster situations and about $89 \%$ success rate in non-disaster environments. The main benefits/features of the proposed handover scheme are briefly highlighted below. The system can self-detect the occurrence of a natural disaster and dynamically adjusts the weights of two parameters namely, DoM and LoP depending on the underlying network environment used. It can also self-predict its own DoM and finally self-select the best suitable TeNB for handover depending on the highest WAS assigned to an eNB.

\section{REFERENCES}

[1] A. Kwasinski, "Analysis of Vulnerabilities of Telecommunication Systems to Natural Disasters, in Proc. of $4^{\text {th }}$ Annual IEEE Systems Conference (SysConf), San Diego, USA, 5-8 April 2010, pp. 359-364.

[2] S. K. Ray, W. Liu, H. Sirisena, D. Deka, S. K. Ray, "An Energy Aware Mobile-Controlled Handover Method for Natural Disaster Situations", in Proc. of $10^{\text {th }}$ Australasian Telecommunication Networks and Applications Conference (ATNAC), Christchurch, New Zealand, 20-22 Nov 2013, pp. 130-135,

[3] T. Fenwick, "Emergency Telephone Call Services and the February 2011 ChCh Earthquake: A Review for the Ministry of Economic Development", August 2011

[4] Y. Nemoto, K. Hamaguchi, "Resilient ICT Research Based on Lessons Learned from the Great East Japan Earthquake" in IEEE Communications Magazine, Vol. 52, Issues 3, pp. 38-43, March 2014

[5] Y. Shibata, N. Uchida, N. Shiratori, "Analysis of and Proposal for a Disaster Information Network from Experience of the Great East Japan Earthquake", in IEEE Communications Magazine, Vol. 52, Issues 3, pp. 44-58, March 2014

[6] J. Blanchard, "Smart Energy Solutions Using Fuel Cells", in IEEE $33^{\text {rd }}$ International Telecommunications Energy Conference (INTELEC), Amsterdam, Netherlands, 9-13 October 2011, pp. 1-8

[7] "Hydrogen Powered Base Stations for Coverage in Disasters and Blackouts", in URL: http://www.gsma.com/publicpolicy/ hydrogenpowered-base-stations-for-coverage-in-disasters-and-blackouts

[8] M. Kobayashi, "Experience of Infrastructure Damage Caused by the Great East Japan Earthquake and Countermeasures against Future Disasters", in IEEE Communications Magazine, Vol. 52, Issues 3, pp. 23-29, March 2014
[9] Y. Sun, K. R. Chowdhury, "Enabling Emergency Communication through a Cognitive Radio Vehicular Network", in IEEE Communications Magazine, Vol. 52, Issue 10, pp. 68-75, October 2014

[10] Q. T. Minh, K. Nguyen, C. Borcea, S. Yamada, "On-the-Fly Establishment of Multihop Wireless Access Networks for Disaster Recovery”, in IEEE Communications Magazine, Vol. 52, Issue 10, pp. 60-66, October 2014

[11] V. S. Rao, "Protocol Signalling Procedures in LTE", White Paper, Radisys Corporation, 2011.

[12] "LTE-Release 9 Technology Introduction", White Paper, Rohde \& Schwarz, 2011

[13] S. K. Ray, "On the design of fast handovers in Mobile WiMAX networks", Ph.D Thesis, University of Canterbury, Christchurch, New Zealand, 2012.

[14] S. K. Ray, S. K. Ray, K. Pawlikowski, A. McInnes, H. Sirisena, "SelfTracking Mobile Station Controls Its Fast Handover in Mobile WiMAX", in Proc. of IEEE Wireless Communications and Networking Conference (WCNC), Sydney, Australia, 18-21 April 2010, pp. 1-6

[15] J. Lorincz, T. Garma and G. Petrovic, "Measurements and Modelling of Base Station Power Consumption under Real Traffic Loads", Sensors Journal, vol. 12, No. 4, pp. 4281-4310, 2012

[16] G. D. L. Roche, A. Glazunov and B. Allen, "LTE-Advanced and NextGeneration Wireless Networks: Channel Modelling and Propagation", Wiley, November 2012 